\title{
Further evidence of the absence of measurable interhemispheric transfer time in left-handers who employ an inverted handwriting posture
}

\author{
WALTER F. McKEEVER \\ Bowling Green State University, Bowling Green, Ohio
}

and

ANNE L. HOFF

Kaiser Hospital, Martinez, California

\begin{abstract}
We previously had found that left-handers who employed the inverted handwriting posture (IHP) showed no significant manual reaction time difference between homolateral and heterolateral stimulus/response-hand conditions, whereas those employing the noninverted handwriting posture (NHP) did (McKeever \& Hoff, 1979). The IHP sinistrals had also shown a pattern of visual-field/response-hand-condition latencies that suggested that they might have a "disconnection" of visual and manual motor areas within the left hemisphere. The results reported here replicate the absence of a homolateral-heterolateral-conditions difference in IHP sinistrals, but not a pattern suggestive of disconnection.
\end{abstract}

Levy (1974) hypothesized that hand posture during writing is diagnostic of several important characteristics of cerebral organization. Thus, inverted handwriting posture (IHP), seen frequently in left-handers but seldom in right-handers, is postulated to indicate predominantly uncrossed pyramidal motor pathways as well as left hemisphere language and right hemisphere visuospatial function lateralities. Left-handers employing a noninverted handwriting posture (NHP) are hypothesized to resemble right-handers in that they are thought to possess crossed motor pathway dominance, but unlike either right-handers or IHP sinistrals, they are postulated to possess right hemisphere language and left hemisphere visuospatial function dominances.

Studies testing the hypothesis of cognitive function laterality differences between IHP and NHP sinistrals have reported generally negative results (see Weber \& Bradshaw, 1981). Studies addressing the hypothesis of differing motor pathway dominances in the two handwriting-posture groups are somewhat more consistent. McKeever and Hoff (1979) and Smith and Moscovitch (1979) found differences in the pattern of visual half-field $\mathrm{x}$ response hand latencies between IHP and NHP groups. The studies differed, however, in the degree of difference obtained. Smith and Moscovitch, who employed a go/no-go manual reaction time (RT) paradigm, found that NHP left-handers and right-

This research was supported by Grant NS 10214-08 from the U.S. Public Health Service (NINCDS). The first author's mailing address is: Department of Psychology, Bowling Green State University, Bowling Green, Ohio 43403. The second author's mailing address is: Kaiser Hospital, Martinez, California 94553. handers both showed faster responses of the hand on the same side as the half-field of dot presentation in a dotdetection paradigm. Such faster homolateral stimulus/ response-hand-condition latencies traditionally have been regarded, since Poffenberger's (1912) experiment, as indicating crossed motor pathway organization. The excess of time required for heterolateral-condition responses over the time required for homolateral-condition responses is taken as a measure of interhemispheric transfer time (ITT). Smith and Moscovitch found that IHP sinistrals had significantly longer homolateralthan heterolateral-condition latencies. This supported Levy's (1974) hypothesis, since ipsilateral motor control of the hands would reverse the usual pattern.

McKeever and Hoff (1979) employed a simple RT experiment in which NHP and IHP left-handers pushed a button on every trial to indicate their detection of light onset following randomly varied intervals between a ready signal and stimulus activation. Like Smith and Moscovitch (1979), they found faster responses to homolateral stimulus/response-hand conditions in NHP persons, a result consistent with crossed motor pathway dominance. For IHP sinistrals, however, they found no difference between the latencies for homolateral and heterolateral conditions. Moreover, there was a pattern of hand $\mathrm{x}$ field interaction that suggested a possible isolation of visual from manual motor areas within the left hemisphere. In left-hand responding, IHP subjects showed left-visual field (LVF) superiority, but they also showed LVF superiority in right-hand responding. By deriving ITT and hemispheric sensory RTs (half-field differences across response hands) from the NHP group data and assuming them to be comparable to those for IHP per- 
sons, McKeever and Hoff (1979) attempted to account for the IHP performances. It was only in right-hand responding that NHP and IHP performance differed. McKeever and Hoff (1979) noted that the large magnitude of the LVF superiority for right-hand responding in the IHP group could be explained by the hypothesis that their left hemisphere areas were isolated from their left hemisphere manual motor-control areas. The time consumed by two crossings of the corpus collosum, one from the left hemisphere to the right hemisphere and then, more anteriorly, from the right hemisphere to left hemisphere motor areas, when added to the excess time required for left as opposed to right hemisphere sensory response, coincided almost perfectly with the obtained LVF-superiority magnitude. Thus, one could hypothesize that IHP sinistrals actually possessed crossed motor control but suffered from a disconnection effect. McKeever and Hoff (1979) noted explicitly that, although the disconnection hypothesis was interesting and could account for the heterolateral-condition superiority in right-hand responding, the hypothesis was purely post hoc and in need of replication.

Finally, a more recent experiment by Bradshaw, Nettleton, and Spehr (1982) found IHP sinistrals to show an ITT comparable to that of right-handers and NHP sinistrals. These investigators employed a choice paradigm, as had Smith and Moscovitch (1979). We noted previously (McKeever \& Hoff, 1979) that the choice paradigm is not well suited to measuring ITT. It obviously involves higher (decisional) processes and is known to be subject to spatial-compatibility effects. The simple RT paradigm does not require decisions and has been shown to be free of spatial-compatibility effects (Anzola, Bertolini, Buchtel, \& Rizzolatti, 1977).

The present experiment was undertaken to provide an additional test of Levy's hypothesis of differing motorpathway organizations in NHP and IHP sinistrals and of the "visual-motor isolation" hypothesis suggested by McKeever and Hoff (1979).

\section{METHOD}

\section{Subjects}

The subjects were 18 IHP left-handers, 12 NHP left-handers, and 12 right-handers. Handedness was defined according to which hand was used for writing. All subjects were volunteers from introductory psychology classes. We selected them according to handedness, but we expressed no interest in handwriting posture until the experiment had been completed. The greater number of IHP subjects reflects the fact that IHP subjects are more common than NHP subjects as defined by our criteria and the decision to have no group with fewer than 12 subjects. Handwriting posture was observed by the experimenters during the subjects' "signing in" prior to the experiment, and this observation permitted accurate counterbalancing with regard to starting hand in the experiment. Handwriting posture was confirmed by formal assessment, following the experiment.

Task and Apparatus

The task was a simple manual response-to-light-onset task. Light stimuli were the standard 4-W fluorescent tubes regularly supplied with Gerbrands tachistoscopes. They were mounted vertically in the forward compartment of a Gerbrands threechannel tachistoscope box, at a distance of $25.0 \mathrm{~cm}$ from the viewing point. They were lateralized $6.5 \mathrm{deg}$ from a small, white, paper fixation circle (1.0 deg in diameter) that was itself centered on a thin, black, vertically positioned strip of paper in the center of the field. The fluorescent tubes were tightly masked at the top and bottom ends by opaque black paper, and Gerbrands plastic light diffusers were positioned in front of the tubes to eliminate "hot" spots. When activated, the tubes produced spatially well-defined illuminations subtending 20.0 deg vertically and $4.5 \mathrm{deg}$ horizontally. The chamber was constantly illuminated by a small incandescent bulb positioned out of view at the top center of the chamber. The luminance of the stimulus positions was $0.6 \mathrm{fL}$ when unlit and $8.5 \mathrm{fL}$ when the stimuli were activated.

Buttonpresses were made on a single button, mounted in the center of an aluminum box, the dimensions of which were $5.7 \times 5.7 \times 12.8 \mathrm{~cm}$ long. Button diameter was $0.8 \mathrm{~cm}$. The box was mounted on a table surface beneath the tachistoscope at midchest height. The long dimension of the button box was oriented parallel to the line of sight. Two box receptacles were employed, one for left-hand responding and one for right-hand responding. These positions were $15.0 \mathrm{~cm}$ apart as measured from button center to button center and corresponded to positions essentially directly forward of the subject's shoulders and $30.0 \mathrm{~cm}$ farther away from him or her than were the stimulus lights. A consistent hand posture was required in pushing the button. All fingers had to be in contact with the box, with the index (response) finger resting on the button and flexed at the first joint.

On each trial, a stimulus light was activated for $100 \mathrm{msec}$. The activation pulse started a Hunter digital-reading millisecond clock, and the subject's response stopped the clock.

\section{Procedure}

The subjects were instructed to focus on the center of the circular fixation point and to avoid moving their eyes or blinking from the time a spoken "ready" signal was given until their response had been made. Coincidently with the ready signal, a switch was closed, which, in turn, activated timers that delayed stimulus-light onsets by 1,2 , or $3 \mathrm{sec}$. These intervals were distributed equally and randomly across visual-half-field (VHF) trials within blocks. Fourteen blocks were administered to each of the subjects. The first 12 blocks contained 24 trials each, and the last 2 blocks contained 12 trials each. Response hand was alternated between blocks within subjects. Starting hand was counterbalanced between subjects within groups. The order of VHF stimulations was fixed within blocks, such that each 3 successive trials were to the same light, with the initially lighted side counterbalanced between blocks. Since the side of stimulus presentation was predictable, it was necessary to monitor the subjects' fixations on all trials. This was done by one experimenter, who used direct visualization of the subjects' eyes through the opened, curtained end of the viewing channel. Trials on which eyeblinks or eye movements occurred (fewer than $1 \%$ of the trials) were discarded. The motivating feedback procedure typically employed in our lab, in which subjects are told their fastest and slowest times at the end of each block and are urged to be as fast as possible, was employed. Three practice trials were given prior to the experimental trials. Three hundred and twelve experimental trials were administered, 156 to each VHF and 78 for each VHF/response-hand combination. Median latencies were computed for each hand-VHF condition for each of the subjects, and these served as the basic scores.

\section{RESULTS}

Our principal interest was in the comparison of response latencies to homolateral and heterolateral 
stimulus-response conditions. This comparison provided the test of crossed versus uncrossed motor pathway dominance. Separate two-factor (hand and field) analyses of variance (ANOVAs) were applied to the data of each group. Subsequently, a one-way ANOVA was applied to the overall mean-median latencies of the three groups to test for a possible difference in overall response speed between groups.

Table 1 presents the basic data of the experiment. Mean-median latencies of the three groups are shown for the various hand and VHF combinations, as well as LVF minus right-visual-field (RVF) latency differences under those conditions. As an aid for the reader, Table 2 presents the mean-median RTs for homolateral and heterolateral conditions directly. These latter figures correspond to the mean-median left-hand/LVF plus righthand/RVF, and left-hand/RVF plus right-hand/LVF latencies, respectively.

The two-way ANOVA for the right-handed group showed a significant main effect of hand $(F=6.28$, $\mathrm{p}<.03$ ), with right-hand responding being faster than left-hand responding (by $3.0 \mathrm{msec}$ ). There was also a trend for faster responses to LVF stimuli $(F=4.29$, $\mathrm{p}<.07)$ in the right-handed group. The interaction of hand and field was significant $(F=5.38, p<.05)$, a result indicative of faster homolateral-condition responses and of crossed motor control of the hands. The NHP sinistral group showed no effect of hand $(F=0.0$, n.s.), no effect of field ( $F=1.18, p>.32)$, and, as in the right-handed group, a significant hand $\mathrm{x}$ field interaction $(F=6.11, p<.03)$. The pattern of homolateral-conditions superiority, and inferred crossed motor-pathway dominance, was thus supported in both right-handed and NHP left-handed subjects. The IHP groups showed significantly faster left- than right-hand responses $(F=$
$7.85, \mathrm{p}<.01)$, no significant field effect $(F=1.44$, $\mathrm{p}>.20)$, and, most critically, no interaction of hand and field factors $(F=0.34, p>.58)$.

A one-way ANOVA comparing the overall RTs of the three groups failed to show any significant difference $(\mathrm{F}=1.38, \mathrm{p}>.27)$. Thus, the somewhat faster overall RT of the IHP group was well within the range of chance variations.

\section{DISCUSSION}

The present results replicate some of our previous findings but not others. The pattern of homolateral-condition superiority obtained for NHP subjects and the inference from it, namely, that NHP left-handers possess contralateral motor control, agrees with previous results. Additionally, the same result has been found for right-handed subjects, a group we had not assessed previously. The result for right-handers provides convergent validity for the task. The absence of a homolateralheterolateral condition difference in IHP sinistrals is consistent with our previous finding for that group as well. In the earlier study, 8 of 15 IHP subjects made faster responses under homolateral conditions, with a mean-median heterolateral superiority (nonsignificant, of course) of $0.6 \mathrm{msec}$ for the entire group. In the present experiment, 9 of 18 IHP subjects were faster under homolateral conditions, with an overall mean-median homolateral superiority of $0.6 \mathrm{msec}$ (also nonsignificant). Both experiments thus showed the clear absence of a result consistent with either ipsilateral or contralateral motor control. The NHP sinistrals in both experiments showed homolateral superiority (10 of 12 and 9 of 12 subjects in the former and present studies, respectively), with significant overall mean-median homolateralcondition-superiority magnitudes of 2.6 and $4.2 \mathrm{msec}$ in the two experiments, respectively.

What was clearly not confirmed was the interesting post hoc hypothesis of the earlier experiment concerning a possible isolation of left hemisphere visual and motor areas in IHP sinistrals. That hypothesis requires LVF superiority comparable to that of the other groups in left-hand responding and an even larger LVF superiority in right-hand responding, with the right-hand/RVF

Table 1

Mean-Median Reaction Times (in Milliseconds) for Response-Hand and Visual-Half-Field Conditions of the Experimental Groups

\begin{tabular}{lcccccc}
\hline & \multicolumn{3}{c}{ Left Hand } & & \multicolumn{3}{c}{ Right Hand } \\
\cline { 2 - 7 } \multicolumn{1}{c}{ Group } & LVF & RVF & LVF-RVF & LVF & RVF & LVF-RVF \\
\hline Right-Handed NHP & 251.8 & 257.3 & -5.5 & 248.3 & 248.7 & -0.4 \\
Left-Handed NHP & 252.1 & 257.8 & -5.7 & 256.2 & 253.4 & +2.8 \\
Left-Handed IHP & 239.2 & 238.6 & +0.6 & 244.8 & 242.8 & +2.0 \\
\hline
\end{tabular}

Table 2

Mean-Median Reaction Times (in Milliseconds) for Homolateral and Heterolateral Stimulus-Response Hand Conditions and Interhemispheric Transfer Time

\begin{tabular}{lcccc}
\hline Group & Homolateral & Heterolateral & Inter- & \\
Conditions & $\begin{array}{c}\text { Transfer } \\
\text { Time }\end{array}$ & $\mathrm{p}$ \\
\hline Right-Handed NHP & 250.3 & 252.8 & +2.5 & .05 \\
Left-Handed NHP & 252.8 & 257.0 & +4.2 & .03 \\
Left-Handed IHP & 241.0 & 241.6 & +0.6 & n.s. \\
\hline
\end{tabular}


condition showing the longest latencies of any condition. In fact, a minuscule RVF superiority was found in left-hand responding, and a somewhat larger RVF superiority was found for right-hand responding. Unlike the situation in our previous experiment, the application of derived ITT and hemispheric sensory-advantage values from either of the other groups cannot account for the performances of the IHP group.

The replicated finding of no measurable ITT for IHP subjects suggests that Levy's (1974) hypothesis is not correct, but that some anomaly of visual-motor integration may exist. The replicated finding of no ITT is open to several conceivable interpretations. One is that the IHP group has no possibility of transferring visual information from one hemisphere to the other. This amounts to the hypothesis that IHP sinistrals may suffer partial or total agenesis of the corpus callosum, with all responses to heterolateral conditions ipsilaterally innervated. This seems an unlikely hypothesis. Since IHP sinistrals are more common than NHP sinistrals, the consistent absence of the corpus callosum would surely have been detected long ago through the procedures of clinical neurology, such as pneumoencephalography or CAT scans. A second hypothesis might be that IHP sinistrals have such efficient interhemispheric transfer that its measurement is beyond the resolving power of the RT procedures. A third, related interpretation might be that visual-motor integration within each hemisphere is somewhat slower than normal and interhemispheric transfer is more efficient than normal.

The ultimate verification of the possible causes of the absence of measurable ITT in IHP subjects must await further studies. What is clearer than it was is that NHP and IHP left-handers do show differences in their patterns of manual response latencies to lateralized visual stimuli, that the performances of IHP sinistrals show no significant ITT, and that this cannot be explained in terms of the visual-motor isolation hypothesis earlier advanced by McKeever and Hoff (1979).

\section{REFERENCES}

Anzola, G. P., Bertolini, G., Buchtel, H. A., \& Rizzolatti,
G. Spatial compatibility and anatomical factors in simple and choice reaction time. Neuropsychologia, 1977, 15, 295-302.

Bradshaw, J. L., Nettleton, N., \& Spehr, K. Sinistral inverters do not possess an anomalous visuomotor organization. Neuropsychologia, 1982, 20, 605-609.

Bradshaw, J. W., \& Taylor, M. J. A word-naming deficit in nonfamilial sinistrals? Laterality effects of vocal responses to tachistoscopically presented letter strings. Neuropsychologia, 1979, 17, 21-32.

LEVY, J. Psychological implications of bilateral asymmetry. In S. Dimond \& J. G. Beaumont (Eds.), Hemisphere function in the human brain. New York: Wiley, 1974.

LEVY, J., \& REID, M. Variations in cerebral organization as a function of handedness, hand posture in writing, and sex. Journal of Experimental Psychology: General, 1978, 107, 119-144.

McKeever, W. F., \& Hoff, A. L. Evidence of a possible isolation of left hemisphere visual and motor areas in sinistrals employing an inverted handwriting posture. Neuropsychologia, $1979,17,445-455$.

McKeever, W. F., \& Van Deventer, A. D. Inverted handwriting position, language laterality, and the Levy-Nagglaki model of handedness and cerebral organization. Neuropsychologia, 1980, 118, 99-102.

Poffenberger, A. T. Reaction time to retinal stimulation with special reference to the time lost in conduction through nerve centers. Archives of Psychology, 1912, 23, 1-73.

Sмıтн, L. C., \& Moscovitch, M. Writing posture, hemispheric control of movement and cerebral dominance in individuals with inverted and noninverted hand postures during writing. Neuropsychologia, 1979, 17, 637-644.

Weber, A. M., \& Bradshaw, J. L. Levy and Reid's neurological model in relation to writing hand posture: An evaluation. Psychological Bulletin, 1981, 89, 74-88.

(Manuscript received for publication April 16, 1983.) 